\title{
Efficient keV X-ray Generation from Irradiation of in-situ Produced Silver Clusters by Ti:sapphire Laser Pulses
}

\author{
U. Chakravarty*, P. A. Naik, S. R. Kumbhare, and P. D. Gupta \\ Laser Plasma Division, Raja Ramanna Centre for Advanced Technology, Indore 452 013, India
}

(Received December 8, 2008 : revised January 30, 2009 : accepted January 30, 2009)

\begin{abstract}
An experimental study of energy absorption and x-ray emission from ultrashort laser pulse irradiation of in-situ produced solid clusters has been performed. Silver clusters produced by a 30 $\mathrm{mJ}, 300 \mathrm{ps}$ laser pulse were irradiated up to an intensity of $3 \times 10^{17} \mathrm{~W} / \mathrm{cm}^{2}$ by a $70 \mathrm{~mJ}, 45 \mathrm{fs}$ compressed laser pulse from the same Ti:sapphire laser. Absorption of the laser light exceeding $70 \%$ was observed, resulting in an x-ray yield $(>1 \mathrm{keV})$ of $\sim 60 \mu \mathrm{J} /$ pulse. This may constitute a much simpler means of intense x-ray generation using ultrashort laser pulses as compared to the irradiation of structured / pre-deposited cluster targets, and it offers higher x-ray conversion efficiency than that from gas clusters and planar solid targets.
\end{abstract}

Keywords: Clusters, Absorption, Femtosecond laser pulses, X-ray emission

OCIS codes : (350.5400) Plasmas; (340.7480) X-rays; (240.0310) Thin films

\section{INTRODUCTION}

In the recent times, there has been a lot of interest in enhancing the absorption of high intensity, ultrashort laser pulses in their interaction with matter [1-3]. The enhanced absorption is manifested in the observation of energetic electrons, $\mathrm{MeV}$ ions and x-rays. The x-rays are useful as a micron sized $\mathrm{x}$-ray source which has many potential applications like time resolved x-ray diffraction studies and x-ray lithography. Since the absorption of the high intensity $\left(\sim 10^{17} \mathrm{~W} / \mathrm{cm}^{2}\right)$ ultrashort laser pulses $(\sim 100 \mathrm{fs})$ by planar solid targets is considerably low ( $\sim 10-20 \%$ at normal incidence) $[4,5]$, various types of targets like gratings [6], structured targets [7], predeposited metal clusters [8], gas clusters [9], snow clusters [10] etc. have been used to enhance the coupling of the laser energy in matter. Increased deposition of the femtosecond pulse energy in the grating targets and in pre-deposited metal clusters, has been achieved through plasmon resonance and field enhancement in protrusions $[6,8]$. However, since the gratings are expensive, they are practically unusable on a routine basis as targets for x-ray sources. Next, high absorption (80-90\%) of ultrashort laser pulses has been observed in gas atom clusters $[11,12]$. The interest in the clusters stems from

*Corresponding author: uday@rrcat.gov.in the fact that the electric field inside the cluster is highly enhanced at three times the critical density $\left(\mathrm{n}_{\mathrm{c}}\right)$ $[13,14]$. Initially, as the clusters absorb the laser energy at near solid density, they get heated up and start expanding. During expansion, the density decreases. When the density approaches $3 \mathrm{n}_{\mathrm{c}}$, the absorption increases rapidly due to the enhancement of the electric field inside the clusters $[9,15]$. However, the $\mathrm{keV}$ x-ray conversion from gas clusters is rather small $(\sim 0.01 \%)$ due to their overall small density [12]. This limits the use of gas atom clusters as an x-ray source. Further, metal clusters have also been used as targets for efficient laser energy absorption. They can be produced by high pressure dc sputtering on polished disks, or by magnetron gas aggregation. They can also be produced by the atom pick-up method. All these methods involve two steps, first depositing the clusters on a planar solid substrate, and then using them as targets for plasma formation [8].

Recently, intense femtosecond laser irradiation of solid targets has been proposed as a simple means of synthesis of clusters of a few tens of nm diameter $[16,17]$. The reason of formation of clusters is the relaxation of material in extreme conditions via fragmentation and molten material ejection [16-18]. We have shown in our earlier experiments large cluster formation (size 10-80nm) using 300 ps laser pulses focused on various materials like $\mathrm{Ag}$, 
In, $\mathrm{Cu}$ etc $[19,20]$, which may be used as targets for $\mathrm{x}$-ray generation. In this paper we present a study of laser energy absorption of intense Ti: Sapphire laser pulses in such in situ produced clusters, and the x-ray generation from them. A high absorption (exceeding $70 \%$ ) of the 45 fs laser pulses is observed at an intensity of $3 \times 10^{17} \mathrm{~W} / \mathrm{cm}^{2}$. Further, an x-ray conversion efficiency (hv $\geq 1 \mathrm{keV}$ ) of $\sim 0.1 \%$ is observed, which is much higher than that achieved from gas clusters or planar solid targets. This suggests that the in-situ produced solid clusters are better targets for x-ray generation than the gas clusters and solids. In addition, the present method offers a simple single-step alternative for $\mathrm{keV} \mathrm{x}$-ray generation compared to the structured targets or pre-deposited clusters.

\section{EXPERIMENTAL SETUP}

In our previous work [19, 20], we have studied cluster generation from solid target irradiation by sub-ns laser pulses from the debris deposition on a substrate (the details of the experimental setup are described in refs 19, 20). SEM pictures of the deposit taken at two prepulse intensities, one at $3 \times 10^{12} \mathrm{~W} / \mathrm{cm}^{2}$ and the other at $10^{10} \mathrm{~W} / \mathrm{cm}^{2}$, clearly revealed a much higher cluster density corresponding to the deposition taken at the higher intensity of the sub-ns pulse. The particle density in this case $\left(\mathrm{I}=3 \times 10^{12} \mathrm{~W} / \mathrm{cm}^{2}\right)$ was estimated to be $\sim 100$ particles $/ \mu \mathrm{m}^{2}$ from the SEM pictures $[19,20]$. In the work reported here, we carried out the absorption measurements of intense femtosecond laser pulses by these in situ produced clusters, using the experimental setup shown in Fig. 1.

The stretched 300 ps laser pulse, before the grating compressor, was split by a pellicle beam splitter and was used to produce silver clusters by focusing it on a planar solid target of silver, in normal incidence geometry. This pulse will be henceforth referred to as the cluster forming "pre-pulse". The focused intensity of the pre-pulse beam was varied between $10^{10}-4 \times 10^{12}$

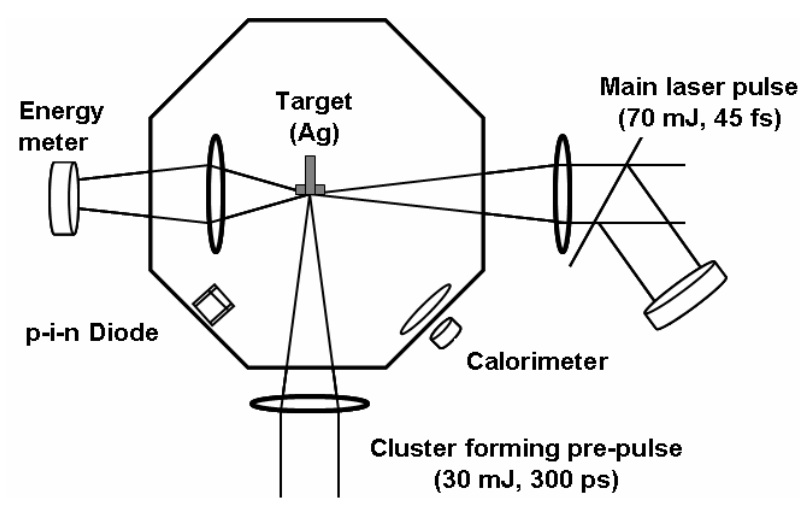

FIG. 1. Schematic of the experimental setup.
$\mathrm{W} / \mathrm{cm}^{2}$. The transmitted part of the uncompressed beam (through the pellicle beam splitter) was time delayed through a delay setup and then compressed to get a pulse of $45 \mathrm{fs}$ duration (referred to as the "main pulse" or the "main beam"). This main beam (70 mJ, $45 \mathrm{fs}$ ) was focused very close to the target, at a distance of $\sim 30 \mu \mathrm{m}$ from the target surface (See Fig.1). This beam propagated parallel to the target surface and irradiated the clusters emanating from the pre-pulse beam irradiating target. For absorption measurements, the main beam energy was measured with and without pre-pulse produced plasma in its path, to get normalized transmission through the plasma plume. After the main beam propagated through the plasma, the transmitted light was collected using a large diameter lens $(75 \mathrm{~mm}$ diameter, $100 \mathrm{~mm}$ focal length) and directed on to a pyro -electric detector (Gentec, sensitivity 3V/J) for energy measurement. Another more sensitive calorimeter (Gentec, sensitivity $164 \mathrm{~V} / \mathrm{J}$ ) was placed outside the chamber to sample the scattered light collected by another lens of focal length $50 \mathrm{~mm}$ placed on the plasma chamber wall window in a direction of $45^{\circ}$ w.r.t. the target. The scattering signal was very low and the angle integrated scattered laser light intensity, normalized to the incident beam, was estimated to be $\leq 1 \%$. Thus, the reduction in transmission of the main pulse beam was predominantly due to its absorption by clusters. Next, an x-ray p-i-n diode (Quantrad) filtered with two aluminized polycarbonate filters having a cut-off (1/e transmission) at $1 \mathrm{keV}$, was used to measure the x-ray radiation emitted by the laser irradiated clusters. The detector subtended a solid angle of $410 \mu$ sr at the source.

\section{RESULTS AND DISCUSSION}

Absorption measurements of the main laser beam were carried out at the maximum main beam intensity of $3 \times 10^{17} \mathrm{~W} / \mathrm{cm}^{2}$, for different intensities of the pre-pulse beam. First, a delay of $10 \mathrm{~ns}$ was kept between the cluster forming pre-pulse and the main pulse. A low absorption was observed as shown in Fig. 2. Despite increase in absorption with pre-pulse energy, the absorption did not increase much $(<10 \%)$ even when the pre-pulse was focused at higher intensities $>10^{12} \mathrm{~W} / \mathrm{cm}^{2}$, where a larger amount of cluster formation is expected. The delay was then increased to 75 ns. From Fig. 2 it is seen that at this delay, there is a low absorption of $\sim 10 \%$ recorded for intensity around $10^{10} \mathrm{~W} / \mathrm{cm}^{2}$. As the pre -pulse intensity is increased, the absorption increases rapidly up to $10^{12}$ $\mathrm{W} / \mathrm{cm}^{2}$, and thereafter, it shows saturation in the range $(1-4) \times 10^{12} \mathrm{~W} / \mathrm{cm}^{2}$, corresponding to a high absorption of $\sim 70 \%$.

The above observations can be understood as follows. At the delay of $10 \mathrm{~ns}$, even at the highest pre-pulse 


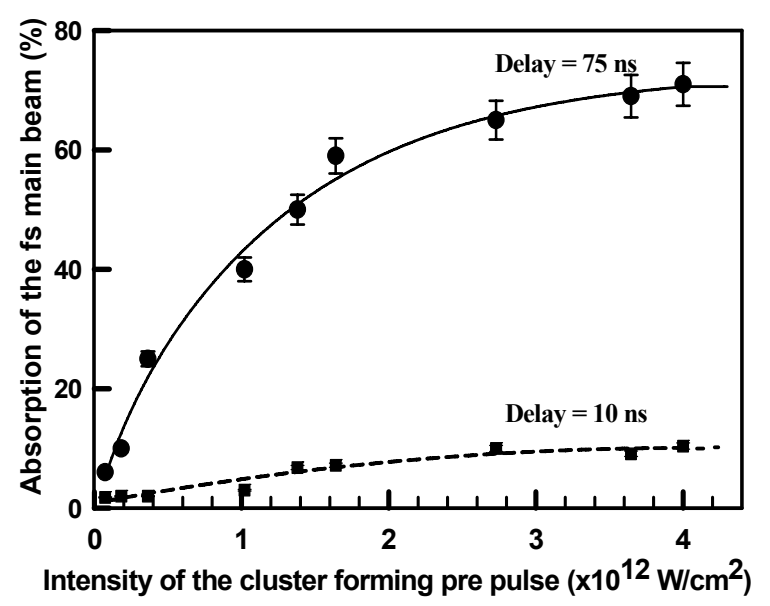

FIG. 2. The variation of the main pulse absorption as a function of the pre-pulse laser intensity at a main beam intensity of $3 \times 10^{17} \mathrm{~W} / \mathrm{cm}^{2}$. The absorption is studied at two delays of $10 \mathrm{~ns}$ and $75 \mathrm{~ns}$ between the pre-pulse and the main pulse.

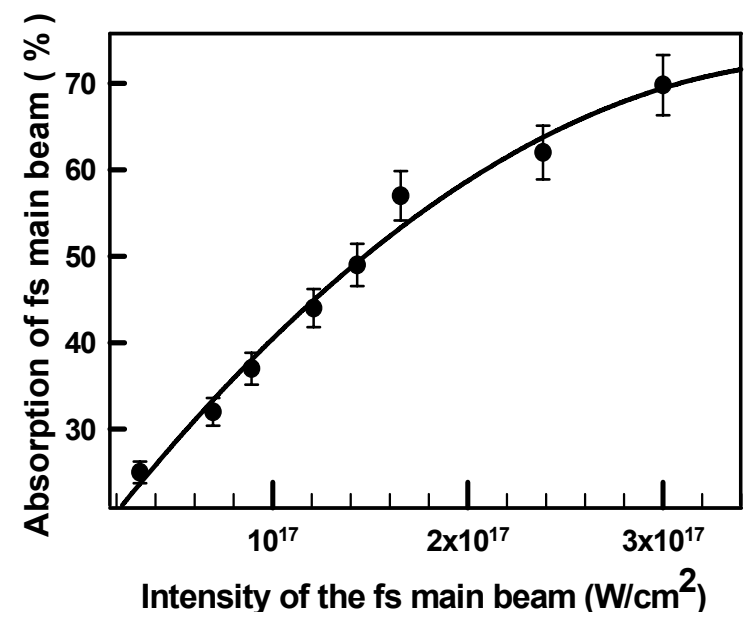

FIG. 3. Variation of the main beam absorption as a function of its intensity. The pre-pulse beam intensity is fixed at $4 \times 10^{12} \mathrm{~W} / \mathrm{cm}^{2}$.

intensity of $4 \times 10^{12} \mathrm{~W} / \mathrm{cm}^{2}$, the large clusters of size $\sim 10$ - $40 \mathrm{~nm}$ (radius) having a typical velocity $\sim 10^{2}-10^{3}$ $\mathrm{m} / \mathrm{s}[21-23]$ will travel a distance of only $\sim 1-10 \mu \mathrm{m}$ from the target surface and hence will not reach the interaction volume of the main pulse, passing $30 \mu \mathrm{m}$ away from the surface. Thus, the main beam will interact only with the plasma produced by the pre-pulse beam (and perhaps some very small clusters traveling with a higher velocity), resulting in the observed low absorption. Increasing the delay results in the arrival of more and more clusters in the interaction region of the main beam. The observed increase in absorption with increasing pre-pulse intensity is then simply understood from the increase in number as well as the velocity of clusters produced at higher pre-pulse intensity $[19,20]$.

Next, the absorption of the main beam was studied

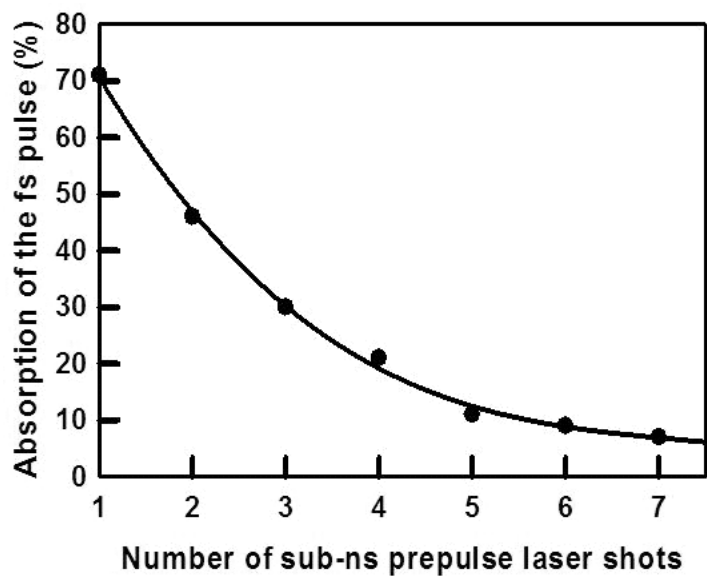

FIG. 4. Variation of the absorption of the main beam with $n^{\text {th }}$ pre-pulse shot fired at the same place in silver target. The fitted curve is only guide to the eyes.

as a function of its intensity, at a fixed pre-pulse intensity of $4 \times 10^{12} \mathrm{~W} / \mathrm{cm}^{2}$. For this purpose, calibrated neutral density filters were used to attenuate the main beam energy, starting from the highest value. The absorption variation for silver target is shown in Fig. 3, where the intensity of the main beam was varied in the range of $3 \times 10^{16}-3 \times 10^{17} \mathrm{~W} / \mathrm{cm}^{2}$. When the intensity was increased by one order of magnitude (from $\sim 3 \times 10^{16}$ $\mathrm{W} / \mathrm{cm}^{2}$ to $3 \times 10^{17} \mathrm{~W} / \mathrm{cm}^{2}$ ), the absorption increased from $\sim 25 \%$ to $70 \%$. The increase in absorption is initially fast and at higher intensities the increase in absorption is slow.

At the fixed pre-pulse beam intensity of $4 \times 10^{12}$ $\mathrm{W} / \mathrm{cm}^{2}$ the increase in absorption with increase in main pulse intensity can also be understood in terms of the $3 \mathrm{n}_{\mathrm{c}}$ resonance absorption in clusters [11,14]. As the clusters absorb the laser energy and get heated up, they start expanding and the density decreases from near solid density. As stated earlier, in the vicinity of $3 \mathrm{n}_{\mathrm{c}}$, a high absorption would occur due to enhancement of laser electric field inside the clusters $[13,14,15]$. For ultrashort laser pulses, this condition will be met more easily at higher laser intensity due to faster expansion of the heated clusters, leading to higher laser energy absorption. Moreover, the rate of change of electron density in a cluster after irradiation is expected to be fast initially for smaller radius (as $\mathrm{dn}_{\mathrm{e}} / \mathrm{dR}$ a $\mathrm{R}^{-4}$ ), hence with increasing intensity of main pulse, a slower change in electron density is expected as most of the expansion occurs during the initial part of the pulse. This explains qualitatively the fast rise in absorption followed by a slower increase of absorption when the main pulse intensity is increased.

It may be relevant to point out that a high absorption was observed only when fresh surface of the target was irradiated. When the pre-pulse laser beam was incident on the previously irradiated spot on the target, the 


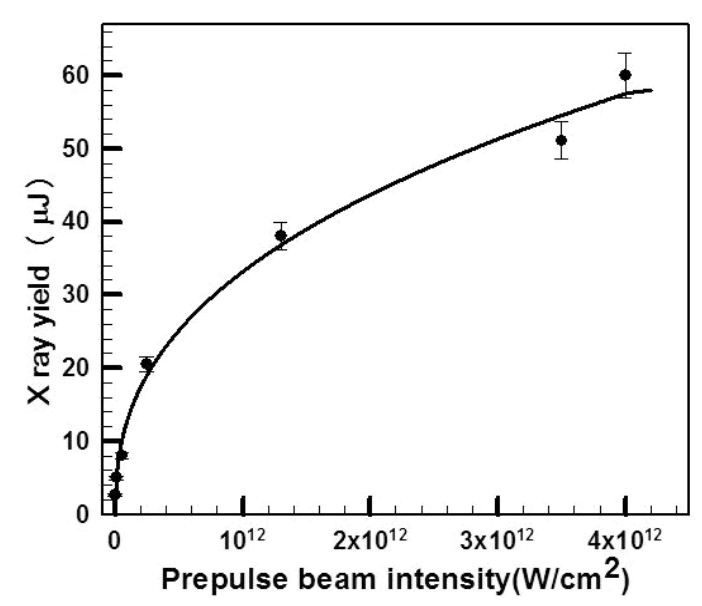

FIG. 5. X-rays emission intensity from silver clusters as a function of the pre-pulse intensity, for main beam intensity of $3 \times 10^{17} \mathrm{~W} / \mathrm{cm}^{2}$.

absorption decreased. Fig. 4 shows the main pulse absorption after a number of pre-pulse shots were fired at the same place. It is seen that the absorption decreases with the number of pre-pulse shots, but it does not reduce to zero.

The above behavior may be understood as follows. When the pre-pulse irradiates a solid surface it forms a crater. On subsequent laser irradiation at the same spot, there will be a recession of the crater surface as more and more material ablates out. Thus the clusters produced from irradiation of the crater region will take a longer time to reach the interaction region and would increasingly miss interaction with the main beam. This inference is also supported from the observation that when the target, after a few shots of irradiation, was brought slightly closer $(\sim 10 \mu \mathrm{m})$ to the main beam, the absorption showed a slight increase compared to that in the unshifted position. There could be a few more reasons for the reduction of absorption. For example, with more laser shots fired at the same place, the focal spot size can increase because of the crater formed by the previous pulses, leading to a decrease in the intensity of the prepulse. Moreover, the modification of the surface structure by the prepulse can also be responsible for the observed decrease in absorption. Next, as stated above, the absorption did not reduce to zero even after a number of shots were fired at the same place. This may be due to arrival of some smaller clusters in the interaction region even after crater formation.

Finally, we present the results of the x-ray emission measurements from the heating of the in-situ produced clusters. Fig. 5 shows the intensity of x-ray emission as a function of the pre-pulse beam intensity at the maximum main beam intensity of $3 \times 10^{17} \mathrm{~W} / \mathrm{cm}^{2}$. It is seen that the $\mathrm{x}$-ray intensity increases with pre-pulse beam intensity. This behavior is consistent with our measurements of a higher absorption of the main beam at high pre-pulse intensity (shown in Fig. 2).

With increase in pre-pulse intensity, the number of clusters interacting with the main pulse increases. As a result, the absorption increases and the x-ray yield also increases, showing a variation somewhat similar to that of the absorption. The spectrally and temporally integrated $\mathrm{x}$-ray yield (for $\mathrm{h} v \geq 1 \mathrm{keV}$ ) at the main beam intensity of $3 \times 10^{17} \mathrm{~W} / \mathrm{cm}^{2}$ and the pre-pulse intensity of $4 \times 10^{12} \mathrm{~W} / \mathrm{cm}^{2}$, was measured to be $\sim 60$ $\mathrm{mJ}$. For the above condition of pre-pulse and the main pulse, the recorded absorption of the high intensity 45 fs pulses was $\sim 70 \%$. This gives a percentage conversion efficiency of the laser energy into $\mathrm{x}$-rays to be $\sim 8.5 \times$ $10^{-2} \%$.

The above high yield of x-rays achieved from silver clusters can be due to several reasons. Irradiation of the metal cluster with intense femtosecond pulses leads to enhanced absorption. Due to the enhanced field inside the clusters, highly charged ion species are produced. Therefore, the clusters give a high x-ray output due to increased collisionality, and higher ionization [24-28]. Due to the above reasons, a high percentage conversion efficiency of laser energy into x-rays is observed. In conventional Xe gas cluster targets, the percentage conversion efficiency of laser energy into $\mathrm{x}$-rays $(\mathrm{h} v>1 \mathrm{keV})$ is reported to be between $1.7-4.2 \times 10^{-3} \%$ [12]. Nickel target in various forms, when irradiated with 1 ps pulse of $10^{17} \mathrm{~W} / \mathrm{cm}^{2}$, produced efficient x-rays above $900 \mathrm{eV}$ [29]. The reported percentage conversion efficiency of $70 \mathrm{~mJ}$ laser energy in to x-rays $(\mathrm{h} v>900 \mathrm{eV})$ from flat nickel target is $2.8 \times 10^{-3} \%$, for Ni nano-groove target it is $2.8 \times 10^{-2} \%$, for $\mathrm{Ni}$ "smoke" targets it is $1.3 \times 10^{-1}$ $\%$, and for $\mathrm{Ni}$ black target it is $1.4 \times 10^{-1} \%$ [29]. This suggests that silver clusters produced in situ are a better source of x-rays than the gas clusters and solids. Moreover, it offers a simple single step method for $\mathrm{keV}$ $\mathrm{x}$-ray generation than the structured targets which are difficult to fabricate.

Next, the temporal duration of the x-ray emission as measured with p-i-n diode lasted for 8 ns (detector limited). While the x-ray emission from the in-situ produced clusters is expected to be isotropic, the debris moves predominantly in the direction of the target normal, in a narrow cone angle. Hence, in a direction parallel to the target surface, there will be very little debris and hence the $\mathrm{x}$-ray source, viewed in this direction, will be nearly debris-free. The present scheme of cluster formation has also the advantage that the interaction of the generated clusters with the main beam is slightly away from the solid target. This prevents heat conduction from clusters to the cold target substrate, leading to enhanced plasma temperature and increased x-ray emission, than in the case of metal clusters pre-deposited on a planar target. 


\section{CONCLUSION}

In conclusion, we have observed a high absorption of $\sim 70 \%$ of high intensity ultrashort laser pulses in silver clusters produced in-situ by a sub-ns pre-pulse. The effect of temporal delay (between the pre-pulse and the main pulse) and the pre-pulse intensity was studied to achieve high absorption of the fs pulses in these clusters. The high laser light absorption resulted in an efficient $\mathrm{keV}$ x-ray generation. A high conversion efficiency of $\sim 8.5 \times 10^{-2} \%$ of the laser energy into $\mathrm{x}$-rays (hv $\geq 1$ $\mathrm{keV}$ ) is observed. Moreover, the $\mathrm{x}$-ray source is practically debris-free.

\section{ACKNOWLEDGMENT}

The authors would like to thank R. A. Khan, B. S. Rao, V. Arora, J. A. Chakera, H. Singhal, and Vikas Kumar for their support during the experiment.

\section{REFERENCES}

1. A. McPherson, B. D. Thomson, A. B. Borisov, K. Boyer, and C. K. Rhodes, "Multiphoton-induced x-ray emission at 4-5 keV from Xe atoms with multiple core vacancies," Nature 370, 631-634 (1994).

2. M. M. Murnane, H. C. Kapteyn, S. P. Gordon, J. Bokor, and E. N. Glytsis, "Efficient coupling of high-intensity subpicosecond laser pulses into solids," Appl. Phys. Lett. 62, 1068-1070 (1993).

3. S. P. Gordon, T. Donnelly, A. Sullivan, H. Hamster, and R. W. Falcone, "X rays from microstructured targets heated by femtosecond lasers,” Opt. Lett. 19, 484-486 (1994).

4. D. F. Price, R. M. More, R. S. Walling, G. Guethlein, R. L. Shepherd, R. E. Stewart, and W. E. White, "Absorption of ultrashort laser pulses by solid targets heated rapidly to temperatures 1-1000 eV," Phys. Rev. Lett. 75, 252-255 (1995).

5. Y. Ping, R. Shepherd, B. F. Lasinski, M. Tabak, H. Chen, H. K. Chung, K. B. Fournier, S. B. Hansen, A. Kemp, D. A. Liedahl, K. Widmann, S. C. Wilks, W. Rozmus, and M. Sherlock, "Absorption of short laser pulses on solid targets in the ultra-relativistic regime," Phys. Rev. Lett. 100, 085004(1-4) (2008).

6. S. Kahaly, S. K. Yadav, W. M. Wang, S. Sengupta, Z. M. Sheng, A. Das, P. K. Kaw, and G. R. Kumar, "Near-complete absorption of intense ultrashort laser light by sub- $\lambda$ gratings,” Phys. Rev. Lett. 101, 145001(1-4) (2008).

7. T. Nishikawa, H. Nakano, K. Oguri, N. Uesugi, K. Nishio, and H. Masuda, "Nanohole-array size dependence of soft $\mathrm{x}$-ray generation enhancement from femtosecond-laserproduced plasma,” J. Appl. Phys. 96, 7537-7534 (2004).

8. P. P. Rajeev, P. Taneja, P. Ayyub, A. S. Sandhu, and G. R. Kumar, "Metal nanoplasmas as bright sources of hard x-ray pulses,” Phys. Rev. Lett. 90, 115002(1-4)
(2003).

9. T. Ditmire, T. Donnelly, A. M. Rubenchik, R. W. Falcone, and M. D. Perry, "Interaction of intense laser pulses with atomic clusters," Phys. Rev. A 53, 3379-3402 (1996).

10. T. Palchan, S. Peckerand, Z. Henis, S. Eisenmann, and A. Zigler, "Efficient coupling of high intensity short laser pulses into snow clusters,” Appl. Phys. Lett. 90, 041501(1-3) (2007).

11. T. Ditmire, R. A. Smith, J. W. G. Tisch, and M. H. R. Hutchinson, "High intensity laser absorption by gases of atomic clusters,” Phys. Rev. Lett. 78, 3121-3124 (1997).

12. T. Ditmire, R. A. Smith, R. S. Marjoribanks, G. Kulcsár, and M. H. R. Hutchinson, "X-ray yields from Xe clusters heated by short pulse high intensity lasers," Appl. Phys. Lett. 71, 166-168 (1997).

13. H. Singhal, V. Arora, P. A. Naik, and P. D. Gupta, "Spectral blue-shifts in laser light scattered from argongas cluster plasmas," Phys. Rev. A 72, 043201(1-7) (2005).

14. J. Zweiback, T. Ditmire, and M. D. Perry, "Femtosecond time-resolved studies of the dynamics of noble-gas cluster explosions," Phys. Rev. A 59, R3166-R3169 (1999).

15. S. Sailaja, R. A. Khan, P. A. Naik, and P. D. Gupta, "Efficient absorption and intense x-ray emission from gas cluster plasmas irradiated by 25 ps laser pulses," IEEE Trans. Plasma Sci. 33, 1006-1012 (2005).

16. S. Amoruso, R. Bruzzese, M. Vitiello, N. N. Nedialkov, and P. A. Atanasov, "Experimental and theoretical investigations of femtosecond laser ablation of aluminum in vacuum,” J. Appl. Phys. 98, 044907(1-7) (2005).

17. S. Eliezer, N. Eliaz, E. Grossman, D. Fisher, I. Gouzman, Z. Henis, S. Pecker, Y. Horovitz, M. Fraenkel, S. Maman, and Y. Lereah, "Synthesis of nanoparticles with femtosecond laser pulses," Phys. Rev. B 69, 144119(1-6) (2004).

18. T. E. Glover, "Hydrodynamics of particle formation following femtosecond laser ablation," J. Opt. Soc. Am. B 20, 125-131 (2003).

19. R. A. Ganeev, U. Chakravarty, P. A. Naik, H. Srivastava, C. Mukherjee, M. K. Tiwari, R. V. Nandedkar, and P. D. Gupta, "Pulsed deposition of metal films and nanoparticles in vacuum using sub-nanosecond laser pulses," Appl. Opt. 46, 1205-1210 (2007).

20. R. A. Ganeev, A. I. Ryasnyanskiy, U. Chakravarty, P. A. Naik, H. Srivastava, M. K. Tiwari, and P. D. Gupta, "Structural, optical, and nonlinear optical properties of indium nanoparticles prepared by laser ablation,” Appl. Phys. B 86, 337-341 (2007).

21. S. Amoruso, R. Bruzzese, N. Spinelli, R. Velotta, M. Vitiello, X. Wang, G. Ausanio, V. Iannotti, and L. Lanotte, "Generation of silicon nanoparticles via femtosecond laser ablation in vacuum,” Appl. Phys. Lett. 84, 4502-4504 (2004).

22. D. Scuderi, O. Albert, D. Moreau, P. P. Pronko, and J. Etchepare, "Interaction of a laser-produced plume with a second time delayed femtosecond pulse," Appl. Phys. Lett. 86, 071502 (1-3) (2005).

23. S. Amoruso, G. Ausanio, A. C. Barone, R. Bruzzese, L. Gragnaniello, M. Vitiello, and X. Wang, "Ultrashort laser ablation of solid matter in vacuum: a comparison between the picosecond and femtosecond regimes," J. Phys. B: Mol. Opt. Phys. 38, L329-L338 (2005). 
24. T. Doppner, Th. Fennel, P. Radcliffe, J. Tiggesbaumker, and K. H. Meiwes-Broer, "Ion and electron emission from silver nanoparticles in intense laser fields," Phys. Rev. A 73, 031202 (1-4) (2006).

25. T. Doppner, Th. Fennel, Th. Diedrich, J. Tiggesbaumker, and K. H. Meiwes-Broer, "Controlling the coulomb explosion of silver clusters by femtosecond dual-pulse laser excitation," Phys. Rev. Lett. 94, 013401(1-4) (2005).

26. T. Ditmire, T. Donnelly, R. W. Falcone, and M. D. Perry, "Strong x-ray emission from high-temperature plasmas produced by intense irradiation of clusters," Phys. Rev. Lett. 75, 3122-3125 (1995).

27. S. Dobosz, M. Lezius, M. Schmidt, P. Meynadier, M.
Perdrix, and D. Normand, J. P. Rozet, and D. Vernhet, "Absolute keV photon yields from ultrashort laser-field -induced hot nanoplasmas," Phys. Rev. A 56, R2526-R2529 (1997).

28. T. Mocek, C. M. Kim, H. J. Shin, D. G. Lee, Y. H. Cha, K. H. Hong, and C. H. Nam, "Enhancement of soft X-ray emission from a cryogenically cooled Ar gas jet irradiated by 25 fs laser pulse," Appl. Phys. Lett. 76, 1819-1821 (2000).

29. G. Kulcsar, D. AlMawlawi, F. W. Bundnik, P. R. Herman, M. Moskovits, L. Zhao, and R. S. Marjoribanks, "Intense picosecond x-ray pulses from laser plasmas by use of nano-structured "Velvet" targets," Phys. Rev. Lett. 84, 5149-5152 (2000). 